\title{
Generalizing the Steiner-Lehmus theorem using the Gröbner cover
}

\author{
Antonio Montes* \\ Universitat Politècnica de Catalunya, Spain. \\ Tomás Recio \\ Universidad de Cantabria, Spain
}

\begin{abstract}
In this note we present an application of a new tool (the Gröbner cover method, to discuss parametric polynomial systems of equations) in the realm of automatic discovery of theorems in elementary geometry. Namely, we describe, through a relevant example, how the Gröbner cover algorithm is particularly well suited to obtain the missing hypotheses for a given geometric statement to hold true. We deal with the following problem: to describe the triangles that have at least two bisectors of equal length. The case of two inner bisectors is the well known, XIXth century old, Steiner-Lehmus theorem, but the general case of inner and outer bisectors has been only recently addressed. We show how the Gröbner cover method automatically provides, while yielding more insight than through any other method, the conditions for a triangle to have two equal bisectors of whatever kind.
\end{abstract}

Keywords: automatic discovery, automatic deduction, elementary geometry, comprehensive Gröbner system, Gröbner cover. 2000 MSC: 13P10, 68T15, 51M04.

cover

\footnotetext{
*Corresponding author

Email addresses: antonio.montes@upc.edu (Antonio Montes), tomas.recio@unican.es (Tomás Recio)

URL: http://www-ma2.upc.edu/ montes/ (Antonio Montes)
} 


\section{Introduction}

In [13] we have introduced and developed the foundations on the application of algorithmic methods for the discussion of parametric polynomial systems of equations to the field of automatic discovery of elementary geometry theorems. The merging of techniques from these two fields (theorem discovery and parametric polynomials) was exemplified through the application of an algorithm for the automatic case-analysis of polynomial systems with parameters (the algorithm $M C C G S$, standing for Minimal Canonical Comprehensive Gröbner System, cf. [10]), to a collection of geometric statements of the kind: Iff $p$, then $q$, where $p$ is only partially known. The automatic discovery protocol allowing such application stems from the work of [16] and has been further extended in [5] and, particularly, in [17]. We refer the interested reader to the above mentioned papers for details and for references to previous and related work.

Now, since the Gröbner cover algorithm, as described in [14], is a substantial improvement of the $M C C G S$ concept and algorithm, it deserved being also tested in a challenging automatic theorem discovery situation, such as the generalization of the Steiner-Lehmus theorem. This is the original goal of this paper.

The theorem of Steiner-Lehmus states that if a triangle has two (internal) angle-bisectors with the same length, then the triangle must be isosceles (the converse is, obviously, also true). This is an issue which has attracted along the years a considerable interest, and we refer to [18] for a large collection of references and comments on this classical statement and its proof. More recently, its generalization, regarding internal as well as external angle bisectors, has been approached through automatic tools, cf. [24], [21] or [1] (related to [2]), where the goal is to find a similar statement concerning triangles verifying the equality of two bisectors (of whatever kind) for different vertices. This generalization has been also achieved through the FSDIC automatic discovery protocol of [5], including the (perhaps new) case describing the simultaneous equality of three (either internal or external) bisectors, placed on each one of the vertices. We refer to [7] (in Spanish) and to [8] for further details on this issue. Moreover, in [9] the case of equality for the internal and external bisectors placed at just one of the vertices of a triangle, has been addressed with automatic discovery tools.

Now, all these results have been obtained through the use of ideal-theoretical elimination methods, which do not allow a fine grain analysis of the in- 
volved situation, in particular, concerning the different behavior of some real-geometry objects, indistinguishable from a complex-geometry point of view, such as the internal/external bisectors at a vertex. We think that the Gröbner cover approach is particularly well suited in this context, bringing out, in its output, the possibility of a detailed case analysis that significantly extends our knowledge of the generalized Steiner-Lehmus theorem.

The paper is organized as follows. Next two Sections briefly outline some basic facts and terminology from Gröbner Covers and from automatic deduction in geometry, respectively. Then, the first part of Section 3 describes the construction of the geometric elements involved in the Steiner-Lehmus statement, its algebraic translation and the Gröbner cover output for the associated parametric system of equations. Subsection 3.1 deals with the geometric interpretation of the obtained results, summarized in Theorem 3.2 (Generalized Steiner-Lehmus theorem). Subsection 3.2 shows the reduction to this context of a closely related problem, obtaining, in this way, a more complete generalization. We end the paper with the section Conclusions, highlighting the contribution of our work.

\section{On Gröbner covers}

There exist different methods to discuss parametric polynomial system of equations that can be used to find new geometrical theorems (some recent ones are $[20,15,6,3])$. We have recently introduced the Gröbner cover (in short: GC) algorithm [14], that gives precise and compact information about parametric polynomial systems of equations. What follows is a short digest on this method.

Let $\bar{a}=a_{1}, \ldots, a_{m}$ be a set of parameters, $\bar{x}=x_{1}, \ldots, x_{n}$ a set of variables and $I \subset K[\bar{a}][\bar{x}]$ an ideal (for example, generated by the set of equations describing a geometric construction, the parameters representing the coordinates of the free points), where $K$ is a computable field (usually $\mathbb{Q}$ ). Denote $\bar{K}$ an algebraically closed extension of $K$ (usually $\mathbb{C}$ ). Then $\bar{K}^{m}$ is the parameter space.

Selecting a monomial order $\succ$ for the variables, the Gröbner cover of $\bar{K}^{m}$ with respect to $I$ is a set of pairs $G C=\left\{\left(S_{i}, B_{i}\right): 1 \leq i \leq s\right\}$, where the $S_{i}$, called segments, are locally closed subsets of the parameter space $\bar{K}^{m}$, and the $B_{i}$ are sets of $I$-regular functions $g_{i j}: S_{i} \rightarrow \mathcal{O}_{S_{i}}[\bar{x}]$ (c.f. [14] for a precise description; the reader can think of them as polynomials, in order to understand what follows), that for every point $a \in S_{i}$ specialize to the 
reduced Gröbner basis of the specialized ideal $I_{a}$, i.e. the ideal obtained from $I$ by evaluating the parameters $\bar{a}$ at point $a$.

Moreover, the segments are disjoint and cover the whole parameter space, the set of leading power products $\left(\operatorname{lpp}_{i}\right)$ of the bases $B_{i}$ on each segment are constant (and characteristic of the segment if the ideal is homogeneous) and the whole description is canonical. The segments depend only on the ideal and the monomial order: they are disjoint, and have a unique reduced Gröbner basis, that is different from the Gröbner basis of the remaining segments. When the system is not homogeneous it can happen that, in dehomogenizing, more than one segment corresponds to the same lpp, but usually in this case the corresponding solutions have different properties. It is known (see [4]) that the set of lpp of the reduced Gröbner basis of a polynomial system characterizes the type of solutions (no solution, finite number of solutions, dimension of the solution set, etc.). Thus, it is natural to attach the information about the $\operatorname{lpp}_{i}$ as a third component of the label associated to the $S_{i}$-segments (even if it is apparent form the $B_{i}$ 's). The Gröbner cover provides, as well, a very compact (i.e. minimal in some sense) discussion of all the involved cases.

There are many different ways of expressing a locally closed set $S$, but for the GC-segments we have chosen a canonical description (the so called P-representation, see [14] for details), consisting of lists of prime ideals of the form

$$
\left.S \rightarrow\left(\mathfrak{p}_{k},\left\{\mathfrak{p}_{k 1}, \ldots, \mathfrak{p}_{k j_{k}}\right\}\right): 1 \leq k \leq t\right)
$$

representing the irreducible components of the Zariski closure of the segment $S$ and of the "holes":

$$
S=\bigcup_{k=1}^{t} \mathbb{V}\left(\mathfrak{p}_{k}\right) \backslash\left(\bigcup_{j=1}^{j_{k}} \mathbb{V}\left(\mathfrak{p}_{k j}\right)\right) .
$$

The $I$-regular functions $g_{i j}$ in the basis $B_{i}$

$$
g_{i j}: S_{i} \rightarrow \mathcal{O}_{S_{i}}[\bar{x}]
$$

are described generically in terms of one polynomial in $\bar{K}[\bar{a}][\bar{x}]$ that specializes (after normalizing) to the corresponding polynomial of the reduced Gröbner basis in (at least) an open subset of the segment; and to zero, in the remaining points of the segment. A generic representation of an I-regular function can be algorithmically extended to a full-representation, consisting on several polynomials such that, for any point in the segment, it exists at least one of them that specializes to non-zero. See [14] for details. 


\section{Automatic discovery of geometric theorems}

Our point of departure is a geometric statement of the kind $\{H \Rightarrow T\}$ (such as: Given a triangle, if we construct the bisectors with respect to the vertices then. . there are at least two bisector segments, from the vertex to the opposite side, of equal length, where $H$ stands for the equations describing the construction (bisector segments) and $T$ describes the desired property (equality of lengths, etc.). By abuse of notation, we will denote also by $H$ and $T$ the ideals generated by the polynomials involved in the equations describing the construction associated to the given statement or the given thesis.

Now, since it is quite reasonable to assume that a given discovery statement is generally false (for instance, not all triangles have two bisectors with equal length), the automatic discovery goal is to search for complementary hypotheses (say, the given triangle should be not degenerate to a line and should be equilateral or isosceles, etc.) providing necessary and sufficient conditions for the thesis to hold.

Although this formulation could seem straightforward, things are quite subtle and involved (for instance, why not to consider the thesis itself as the only needed complementary hypotheses?). Therefore, as stated in the Introduction, there is a variety of protocols (precise formulation of goals and algorithmic procedures to achieve them) concerning the automatic discovery of geometric theorems. Among them, those of [21], [16], [5], are-grosso modofounded in ideal theoretic elimination theory, searching for a single conjunction of equations and negated equations as the complementary hypotheses (that is, the difference of two algebraic sets).

On the other hand, the approaches of [13] and [17] allow -roughly speakingworking with more general constructible sets, ie. finding a finite union of collections of equations $R_{i}^{\prime}$ in the parameters, and inequalities $R_{i}^{\prime \prime}$ (some of them in the parameters, to take care of the possible degenerate cases of the free variables for the given construction, and some in a subset of variables from these parameters, to consider the possible degenerate cases after including the new hypotheses $R_{i}^{\prime}$ ), which would provide

- when added to $H$, sufficient conditions for $T$, so that

$$
\left\{\left(H \wedge\left(\vee_{i}\left(R_{i}^{\prime} \wedge \neg R_{i}^{\prime \prime}\right)\right)\right) \Rightarrow T\right\}
$$

- which are as well necessary, so that $\left\{(T \wedge H) \Rightarrow\left(H \wedge\left(\vee_{i}\left(R_{i}^{\prime} \wedge \neg R_{i}^{\prime \prime}\right)\right)\right)\right\}$ 
Therefore, as argued in detail in [13] and [17], a reasonable way to proceed in order to find a collection of polynomials $R_{i}^{\prime}, R_{i}^{\prime \prime}$ verifying the above conditions could consist in computing the projection over the parameter space of the solution set of all hypotheses and theses equations, $V(H) \cap V(T)$, and express it as $\bigcup_{i}\left(V\left(R_{i}^{\prime}\right) \backslash V\left(R_{i}^{\prime \prime}\right)\right)$. Yet, we should check if over each component of the union, the corresponding set of equations and inequations yield sufficient conditions for $T$.

In practice, this could be achieved as follows. First, consider a geometrical construction depending on a set of points $\bar{A}=\left\{A_{1}, \ldots, A_{s}\right\}$, whose free coordinates are taken as parameters $\bar{a}$. The construction produces some new dependent points $\bar{P}=\left\{P_{1}, \ldots, P_{r}\right\}$, whose coordinates are taken as dependent variables $\bar{x}$.

The problem is determining the configuration of the points $\bar{A}$, the parameters $\bar{a}$ varying in the parameter space $\mathbb{C}^{m}$, in order that the points $\bar{P}$ verify some property (for example, they are the end points of the bisectors with equal length). For this purpose, we write the equations reflecting the geometric construction and the theses, and we consider the corresponding parametric ideal $I \subset \mathbb{Q}[\bar{a}][\bar{x}]$.

Let $\left\{\left(S_{i}, B_{i}\right): 1 \leq i \leq s\right\}$ be the Gröbner cover of the parameter space wrt to $I$. Then we will have to carefully analyze its output, bearing in mind that

- As the locus of free points where the theorem holds should -when the given statement is not generally true, which is the usual case for discovery- have dimension less than the whole parameter space, the only open segment in the GC (also called the generic segment) must correspond to $\operatorname{lpp}=\{1\}$. Thus, the generic segment will be of the form

$$
S_{1}=\bar{K}^{m} \backslash \bigcup_{i} \mathbb{V}\left(\mathfrak{p}_{i}\right)
$$

- The remaining segments will be all inside $\bigcup_{i} V\left(\mathfrak{p}_{i}\right)$

- If the points $P_{i}$ are uniquely determined by the points $A_{j}$, we will find a segment $S_{2}$ corresponding to a single solution in $\bar{x}$ with reduced Gröbner basis having the full set of coordinates as lpp.

- There can be segments lifting up to more than one solution, that we have then to analyze in detail. 
- There can also exist segments corresponding to degenerate or lifting up to non-real constructions in which we are, in general, less interested.

The important fact about the use of Gröbner Cover in this context is that it provides -in a compact and concise way- all the essential pieces (a finite number of them) on the parameter space, allowing to determine those that correspond to the validity of the given statement.

\section{Steiner-Lehmus theorem}

To show the power of the outlined procedure, we will apply it to find a generalization of the Steiner-Lehmus Theorem. This theorem was proposed by the well known, XIXth century geometer, Steiner, to Sturm, and it was proved by Lehmus for the first time in 1848. It could be stated as follows:

Theorem 3.1 (Classical Steiner-Lehmus). The inner bisectors of angles $A$ and $B$ of a triangle $A B C(\alpha=\beta$ and $\gamma=\delta)$ are of equal length $\left(\overline{A A^{\prime}}=\overline{B B^{\prime}}\right)$ if and only if the triangle is isosceles with $\overline{A C}=\overline{B C}$ (see Figure 1).

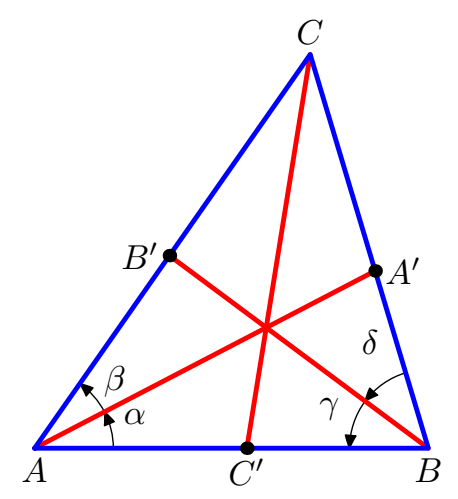

Figure 1: Triangle and inner bisectors

We refer the reader to the Introduction concerning recent work on this statement from the point of view of automatic theorem proving and discovery.

Now, in order to automatically discover the Steiner-Lehmus theorem with GC, let $A B C$ be the given triangle and consider the bisectors at angle $A$. To 
construct the bisectors (see Figure 2) we consider the circle with center A and radius $\overline{A C}$. There are two intersection points $P$ and $P^{\prime}$ of the circle with side $A B$, and thus two middle points $Q$ and $Q^{\prime}$ of $\overline{C P}$ and $\overline{C P^{\prime}}$ determining the bisectors $\overline{A M}$ and $\overline{A M^{\prime}}$ whose length we are interested in. So, if we only use the equations determining $M$ and $M^{\prime}$ we will not distinguish between the inner and the outer bisector. It is precisely this difficulty that will allow to generalize the theorem.

Without loss of generality, we set coordinates $A(0,0), B(1,0), C(a, b)$. Then let $(p, 0)$ be the intersection of the circle centered at $A$ passing through $C$, (i.e. points $P$ or $\left.P^{\prime}\right)$, and let $\left(x_{1}, y_{1}\right)$ stand for the feet of the bisectors, (i.e. points $M$ or $\left.M^{\prime}\right)$. The equation of the circle is $\left(a^{2}+b^{2}\right)-p^{2}$. The middle point between $(0, p)$ and $C$ is $Q=\left(\frac{a+p}{2}, \frac{b}{2}\right)$. Point $\left(x_{1}, y_{1}\right)$ is on the line $A Q$ and so $b x_{1}-(a+p) y_{1}$ expresses that $\left(x_{1}, y_{1}\right)$ is on the bisector line. Finally the equation staying that $\left(x_{1}, y_{1}\right)$ lies on side $B C$, is $b\left(1-x_{1}\right)+(a-1) y_{1}$. Thus, the equations determining $\left(x_{1}, y_{1}\right)$ in terms of $(a, b)$ are:

$$
\left(a^{2}+b^{2}\right)-p^{2}, b x_{1}-(a+p) y_{1}, b\left(1-x_{1}\right)+(a-1) y_{1} .
$$

Notice the sign of $p$ discriminates which bisector of $A$ is being concerned with. If a solution of our problem has $p>0$ it will correspond to the inner bisector of $A$, whereas a solution with $p<0$ will correspond to the outer bisector of $A$. But the sign is not algebraically (from the complex point of view) relevant, so both points $M$ and $M^{\prime}$ are solutions of the same equations. The length of the bisector is $l_{A}^{2}=x_{1}^{2}+y_{1}^{2}$.

Consider now the bisectors of $B$ (see Figure 2). Denoting $(r, 0)$ the intersection point of the circle centered in $B$ with radius $\overline{B C}$ (points $R$ or $R^{\prime}$ ) and $\left(x_{2}, y_{2}\right)$ the coordinates of the foot of the bisector of $B$ (points $T$ or $T^{\prime}$ ) the corresponding equations for them are:

$$
(a-1)^{2}+b^{2}-(r-1)^{2},\left(1-x_{2}\right) b+(a+r-2) y_{2}, a y_{2}-b x_{2} .
$$

In that case, a discriminator between inner and outer bisectors of $B$ is $1-r$. A solution with $1-r>0$ will correspond to the inner bisector whereas a solution with $1-r<0$ will correspond to the outer bisector. The length of the bisector of the angle $B$ is $l_{B}^{2}=\left(x_{2}-1\right)^{2}+y_{2}^{2}$.

Now, using the set of all the above equations, we turn to searching the necessary and also sufficient conditions for assuring that the length of one bisector of the angle $A$ is equal to that of one bisector of angle $B$ (our Problem 1, we will later address the analogous problem for vertices $A, C$ and 


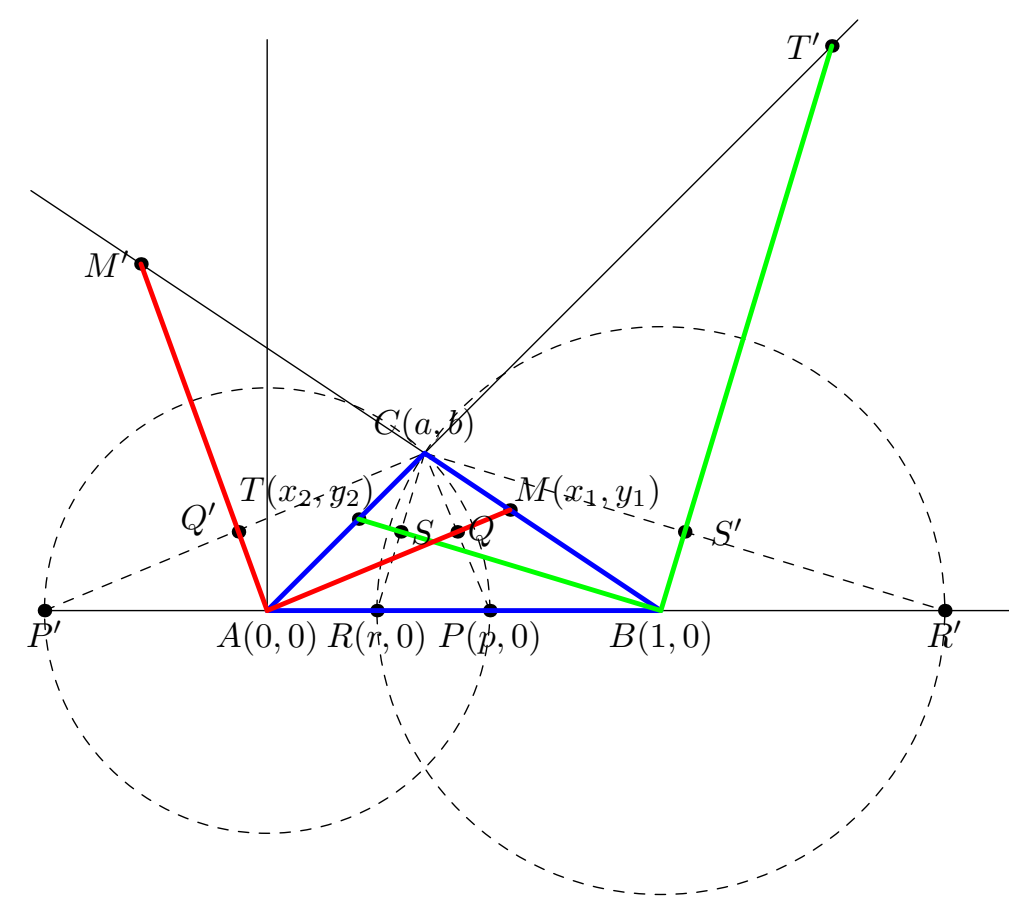

Figure 2: The bisectors of $A$ and $B$ are equal

$B, C)$, but we are not distinguishing between which inner or outer bisector is concerned. It can happen that the two equal bisectors are the two inner bisectors $\left(i_{A}=i_{B}\right)$, or the two outer bisectors $\left(e_{A}=e_{B}\right)$, or one inner and one outer bisector (cases $i_{A}=e_{B}$ and $e_{A}=i_{B}$ ). There are, thus, four possibilities.

In order to compute the Gröbner cover, we include the set of equations (1), the set of equations (2), plus the condition that the length of one bisector of $A$ is equal to that of one bisector of $B$, i.e. $x_{1}^{2}+y_{1}^{2}=\left(x_{2}-1\right)^{2}+y_{2}^{2}$. Thus 
the complete set of equations is:

$$
\left\{\begin{array}{l}
a^{2}+b^{2}-p^{2} \\
b x_{1}-(a+p) y_{1} \\
b\left(1-x_{1}\right)+(a-1) y_{1} \\
(a-1)^{2}+b^{2}-(r-1)^{2} \\
b\left(1-x_{2}\right)+(a+r-2) y_{2} \\
a y_{2}-b x_{2} \\
x_{1}^{2}+y_{1}^{2}=\left(x_{2}-1\right)^{2}+y_{2}^{2}
\end{array}\right.
$$

Now, we take the point $C(a, b)$ as the only parametric point, for which we want to obtain the conditions for the system (3) with variables $x_{1}, y_{1}, x_{2}, y_{2}, p, r$ to have solutions. These solutions will correspond to one bisector of $A$ being equal to one bisector of $B$, but the conditions over $a, b$ will not distinguish between internal and external bisectors. When $p$ is positive, the bisector of $A$ will be internal and it will be external if $p$ is negative. The same happens considering the sign of $1-r$, for the bisector of $B$.

The GC algorithm is used here taking the grevlex $\left(x_{1}, y_{1}, x_{2}, y_{2}, p, r\right)$ order for the variables. The call in Singular (after charging the grobcov library) is:

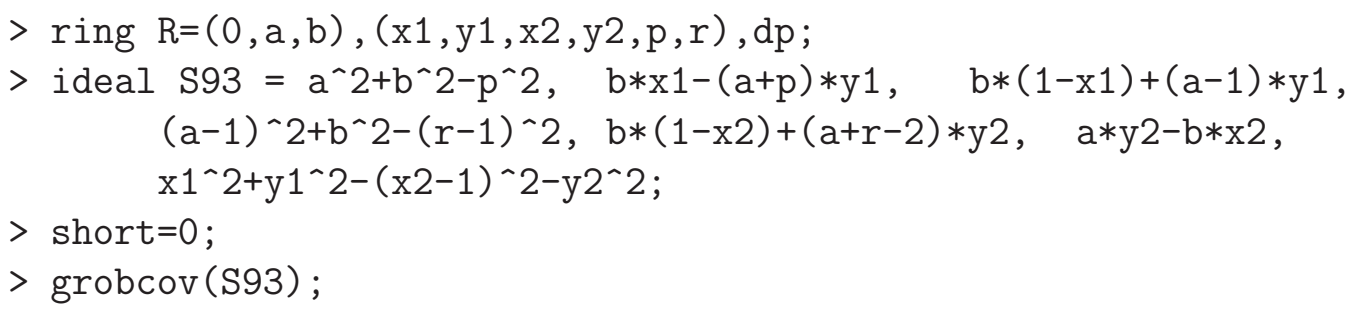

Let us describe below and in the following tables the output of the Gröbner cover algorithm. The following irreducible curves and varieties (over Q) appear:

$$
\begin{aligned}
\mathcal{C}_{1}= & \mathbb{V}\left(8 a^{10}-40 a^{9}+41 a^{8} b^{2}+76 a^{8}-164 a^{7} b^{2}-64 a^{7}\right. \\
& +84 a^{6} b^{4}+246 a^{6} b^{2}+16 a^{6}-252 a^{5} b^{4}-164 a^{5} b^{2} \\
& +8 a^{5}+86 a^{4} b^{6}+278 a^{4} b^{4}+31 a^{4} b^{2}-4 a^{4}-172 a^{3} b^{6} \\
& -136 a^{3} b^{4}+20 a^{3} b^{2}+44 a^{2} b^{8}+122 a^{2} b^{6}+14 a^{2} b^{4} \\
& -10 a^{2} b^{2}-44 a b^{8}-36 a b^{6}+12 a b^{4}+9 b^{10}+14 b^{8} \\
& -b^{6}-6 b^{4}+b^{2}, \\
\mathcal{C}_{2}= & \mathbb{V}(2 a-1) . \\
\mathcal{C}_{3}= & \mathbb{V}(b),
\end{aligned}
$$


We are interested only in the real points, so we separate the real from the complex points appearing in the segments.

\begin{tabular}{|c|c|c|}
\hline Varieties & & Real points \\
\hline$V_{1}=\mathbb{V}(b, a)$ & $P_{1}$ & $=(0,0)$ \\
\hline$V_{2}=\mathbb{V}(b, a-1)$ & $P_{2}$ & $=(1,0)$ \\
\hline$V_{3}=\mathbb{V}\left(b, 2 a^{2}-2 a-1\right)$ & $\begin{array}{l}P_{31} \\
P_{32}\end{array}$ & $\begin{array}{l}=\left(\frac{1-\sqrt{3}}{2}, 0\right) \\
=(-.3660254038,0 .) \\
=\left(\frac{1+\sqrt{3}}{2}, 0\right) \\
=(1.366025404,0 .)\end{array}$ \\
\hline$V_{4}=\mathbb{V}(b, 2 a-1)$ & $P_{4}$ & $=\left(\frac{1}{2}, 0\right)$ \\
\hline$V_{5}=\mathbb{V}\left(12 b^{2}-1,2 a-1\right)$ & $\begin{array}{l}P_{51} \\
P_{52}\end{array}$ & $\begin{array}{l}=\left(\frac{1}{2},-\frac{\sqrt{3}}{6}\right) \\
=(0.5,-0.2886751347) \\
=\left(\frac{1}{2}, \frac{\sqrt{3}}{6}\right) \\
=(0.5,0.2886751347)\end{array}$ \\
\hline$V_{6}=\mathbb{V}\left(4 b^{2}-3,2 a-1\right)$ & $\begin{array}{l}P_{61} \\
P_{62}\end{array}$ & $\begin{array}{l}=\left(\frac{1}{2},-\frac{\sqrt{3}}{2}\right) \\
=(.5000000000,-.8660254040) \\
=\left(\frac{1}{2}, \frac{\sqrt{3}}{2}\right) \\
=(0.5, .8660254040)\end{array}$ \\
\hline$V_{7}=\mathbb{V}\left(b^{4}+11 b^{2}-1,5 a-2 b^{2}-6\right)$ & $\begin{array}{l}P_{71} \\
P_{72}\end{array}$ & $\begin{array}{l}=\left(-1+\sqrt{5},-\frac{\sqrt{-22+10 \sqrt{5}}}{2}\right) \\
=(1.236067977,-.3002831039) \\
=\left(-1+\sqrt{5}, \frac{\sqrt{-22+10 \sqrt{5}}}{2}\right) \\
=(1.236067977, .3002831039)\end{array}$ \\
\hline$V_{8}=\mathbb{V}\left(b^{4}+11 b^{2}-1,5 a+2 b^{2}+1\right)$ & $\begin{array}{l}P_{81} \\
P_{82}\end{array}$ & $\begin{array}{l}=\left(2-\sqrt{5},-\frac{\sqrt{-22+10 \sqrt{5}}}{2}\right) \\
(-.236067977,-.3002831039) \\
\left(2-\sqrt{5}, \frac{\sqrt{-22+10 \sqrt{5}}}{2}\right) \\
(-.236067977, .3002831039)\end{array}$ \\
\hline$V_{9}=\mathbb{V}\left(4 b^{4}+5 b^{2}+2,2 a-1\right)$ & & \\
\hline
\end{tabular}




\begin{tabular}{|c|ll|}
\hline Vars. & Complex points & \\
\hline$V_{7}$ & $P_{73}=\left(-1-\sqrt{5},-I \frac{\sqrt{22+10 \sqrt{5}}}{2}\right)$ & $=(-3.236067977,-3.330190676 I)$ \\
& $P_{74}=\left(-1+\sqrt{5}, I \frac{\sqrt{22+10 \sqrt{5}}}{2}\right)$ & $=(-3.236067977,3.330190676 I)$ \\
\hline$V_{8}$ & $P_{83}=\left(2+\sqrt{5},-I \frac{\sqrt{22+10 \sqrt{5}}}{2}\right)$ & $=(4.236067977,-3.330190676 I)$ \\
& $P_{84}=\left(2+\sqrt{5}, I \frac{\sqrt{22+10 \sqrt{5}}}{2}\right)$ & $=(4.236067977,3.330190676 I)$ \\
\hline$V_{9}$ & $P_{91}=\left(\frac{1}{2},-\frac{\sqrt{-10+2 I \sqrt{7}}}{4}\right)$ & $=(0.5,-.2026163631-.8161209412 I)$ \\
& $P_{92}=\left(\frac{1}{2}, \frac{\sqrt{-10+2 I \sqrt{7}}}{4}\right)$ & $=(0.5,0.2026163631+.8161209412 I)$ \\
& $P_{93}=\left(\frac{1}{2},-\frac{\sqrt{-10-2 I \sqrt{7}}}{4}\right)$ & $=(0.5,-0.2026163631+.8161209412 I)$ \\
& $P_{94}=\left(\frac{1}{2}, \frac{\sqrt{-10-2 I \sqrt{7}}}{4}\right)$ & $=(0.5,0.2026163631-.8161209412 I)$ \\
\hline
\end{tabular}

These curves are represented in Figure 3. Special points are either singular points of $\mathcal{C}_{1}$ or intersection points between the three curves:

a) $V_{1}, V_{2}, V_{5}, V_{7}, V_{8}$ are singular points of $\mathcal{C}_{1}$. They contain the real points $P_{1}, P_{2}, P_{51}, P_{52}, P_{71}, P_{72}, P_{81}, P_{82}$ and some other complex points.

b) $V_{5}, V_{6}, V_{9}$ are intersection points between $\mathcal{C}_{1}$ and $\mathcal{C}_{2}$. They contain the real points $P_{51}, P_{52}, P_{61}, P_{62}$ plus other complex points.

c) $V_{1}, V_{2}$ are intersection points between $\mathcal{C}_{1}$ and $\mathcal{C}_{3}$. They contain the real points $P_{1}=A$ and $P_{2}=B$.

d) $V_{3}$ is the intersection between $\mathcal{C}_{2}$ and $\mathcal{C}_{3}$.

Variety $V_{9}$ contains only complex points, whereas $V_{7}$ and $V_{8}$ contain real and complex points. We distinguish both cases because of the particular behavior of complex points concerning Euclidean distance issues and because we are not interested in the complex points.

Let us give now the output of the Gröbner cover. We obtain the following description with 9 segments: 
1. Segment with $\operatorname{lpp}=\{1\}$

Segment: $\mathbb{C}^{2} \backslash\left(\mathcal{C}_{1} \cup \mathcal{C}_{2} \cup \mathcal{C}_{3}\right)$

Generic segment

Description: The whole parameter space except the curves $\left(\mathcal{C}_{1} \cup \mathcal{C}_{2} \cup \mathcal{C}_{3}\right)$. Basis: $B_{1}=\{1\}$ (no solution over the segment)

2. Segment with $\operatorname{lpp}=\left\{p, y_{2}, x_{2}, y_{1}, x_{1}, r^{2}\right\}$

Segment: $\left(\mathcal{C}_{2} \backslash\left(V_{4} \cup V_{5} \cup V_{6}\right)\right) \cup V_{8}$

Description: $\left(\mathcal{C}_{2}\right.$ minus intersecting points with $\mathcal{C}_{1}$ and $\left.\mathcal{C}_{2}\right)$ plus $V_{8}$

Basis: (2 solutions on each point of the segment)

$$
\begin{aligned}
B_{2}= & \left\{(35 a-45) p+\left(-4 a b^{2}-37 a+2 b^{2}-9\right) r+(65 a-5)\right. \\
& \left(a-2 b^{2}+1\right) y_{2}+(-4 a b) r, \quad\left(7 a+2 b^{2}-5\right) x_{2}+(-2 a+2) r \\
& \left(100 a b^{3}-75 a b+60 b^{3}-45 b\right) y_{1}+\left(-28 a b^{2}+16 a+124 b^{2}-8\right) r \\
& +\left(-940 a b^{2}+80 a+470 b^{2}-40\right),\left(220 b^{2}-165\right) x_{1} \\
& +\left(-16 a b^{2}-148 a+8 b^{2}-36\right) r+\left(160 a b^{2}+380 a-300 b^{2}-25\right), \\
& \left.(4 a) r^{2}+(-8 a) r+\left(a-2 b^{2}+1\right)\right\}
\end{aligned}
$$

3. Segment with $\operatorname{lpp}=\left\{r, p, y_{2}, x_{2}, y_{1}, x_{1}\right\}$

Segment: $\mathcal{C}_{1} \backslash\left(V_{1} \cup V_{2} \cup V_{3} \cup V_{5} \cup V_{6} \cup V_{7} \cup V_{8} \cup V_{9}\right)$

Description of the real points: The curve $\mathcal{C}_{1}$ except the points $P_{1}, P_{2}, P_{31}, P_{32}, P_{51}, P_{52}, P_{61}, P_{62}, P_{71}, P_{72}, P_{81}, P_{82}$

Basis: (single solution on each point of the segment)

$$
\begin{aligned}
B_{3}= & \left\{\left(3 a^{4}-6 a^{3}+6 a^{2} b^{2}+5 a^{2}-6 a b^{2}+3 b^{4}+5 b^{2}-1\right) r+\left(a^{5}-10 a^{4}\right.\right. \\
& \left.+2 a^{3} b^{2}+17 a^{3}-18 a^{2} b^{2}-10 a^{2}+a b^{4}+17 a b^{2}-a-8 b^{4}-10 b^{2}+2\right), \\
& \left(3 a^{4}-6 a^{3}+6 a^{2} b^{2}+5 a^{2}-6 a b^{2}-4 a+3 b^{4}+5 b^{2}+1\right) p \\
& +\left(a^{5}+2 a^{4}+2 a^{3} b^{2}-7 a^{3}+6 a^{2} b^{2}+4 a^{2}+a b^{4}-7 a b^{2}-a+4 b^{4}+4 b^{2}\right), \\
& \left(a^{5}-4 a^{4}+2 a^{3} b^{2}+5 a^{3}-6 a^{2} b^{2}+a b^{4}+5 a b^{2}-a-2 b^{4}\right) y_{2} \\
& +\left(-3 a^{4} b+6 a^{3} b-6 a^{2} b^{3}-5 a^{2} b+6 a b^{3}-3 b^{5}-5 b^{3}+b\right), \\
& \left(a^{5}-4 a^{4}+2 a^{3} b^{2}+5 a^{3}-6 a^{2} b^{2}+a b^{4}+5 a b^{2}-a-2 b^{4}\right) x_{2} \\
& +\left(-3 a^{5}+6 a^{4}-6 a^{3} b^{2}-5 a^{3}+6 a^{2} b^{2}-3 a b^{4}-5 a b^{2}+a\right), \\
& \left(a^{5}-a^{4}+2 a^{3} b^{2}-a^{3}-a^{2}+a b^{4}-a b^{2}+3 a+b^{4}-b^{2}-1\right) y_{1} \\
& +\left(3 a^{4} b-6 a^{3} b+6 a^{2} b^{3}+5 a^{2} b-6 a b^{3}-4 a b+3 b^{5}+5 b^{3}+b\right), \\
& \left(a^{5}-a^{4}+2 a^{3} b^{2}-a^{3}-a^{2}+a b^{4}-a b^{2}+3 a+b^{4}-b^{2}-1\right) x_{1} \\
& +\left(2 a^{5}-8 a^{4}+4 a^{3} b^{2}+12 a^{3}-12 a^{2} b^{2}-8 a^{2}+2 a b^{4}+12 a b^{2}+2 a\right. \\
& \left.\left.-4 b^{4}-4 b^{2}\right)\right\}
\end{aligned}
$$


4. Segment with $\operatorname{lpp}=\left\{y_{2}, y_{1}, r^{2}, p^{2}, x_{1}^{2}\right\}$

Segment: $\mathcal{C}_{3} \backslash\left(V_{1} \cup V_{2}\right)$

Description: The line $\mathcal{C}_{3}$ except the points $P_{1}, P_{2}$

Basis: (infinite solutions, corresponding to degenerate triangles)

$$
\begin{aligned}
B_{4}= & \left\{y_{2}, y_{1}, r^{2}-2 r+\left(-a^{2}+2 a\right), p^{2}+\left(-a^{2}\right)\right. \\
& \left.x_{1}^{2}-x_{2}^{2}+2 x_{2}-1\right\}
\end{aligned}
$$

5. Segment with $\operatorname{lpp}=\left\{y_{2}, x_{2}, y_{1}, x_{1}, r^{2}, p^{2}\right\}$

Segment: $V_{5}$

Description: Points $P_{51}, P_{52}$

Basis: (4 solutions on each point of the segment)

$$
\begin{aligned}
B_{5}= & \left\{2 y_{2}-3 b r, 4 x_{2}-3 r, 2 y_{1}+3 b p-3 b,\right. \\
& \left.4 x_{1}-3 p-1,3 r^{2}-6 r+2,3 p^{2}-1\right\}
\end{aligned}
$$

6. Segment with $\operatorname{lpp}=\left\{r, p, y_{2}, x_{2}, y_{1}, x_{1}\right\}$

Segment: $V_{6}$

Description: Points $P_{61}, P_{62}$

Basis: (single solution on the points of the segment)

$$
B_{6}=\left\{r, p-1,2 y_{2}-b, 4 x_{2}-1,2 x_{1}-b, 4 x_{1}-3\right\}
$$

7. Segment with lpp $=\left\{r, y_{2}, x_{2}, y_{1}, x_{1}, p^{2}\right\}$

Segment: $V_{7}$

Description: Points $P_{71}, P_{72}$

Basis: (2 solutions on each point of the segment)

$$
\begin{aligned}
B_{7}= & \left\{5 r+\left(b^{2}-7\right),(5 b) y_{2}+\left(3 b^{2}-1\right), x_{2}-2,\right. \\
& (5 b) y_{1}+\left(3 b^{2}-1\right) p+\left(-3 b^{2}+1\right), \\
& \left.5 x_{1}+\left(b^{2}-2\right) p+\left(-b^{2}-3\right), 5 p^{2}+\left(-b^{2}-8\right)\right\} .
\end{aligned}
$$

8. Segment with $\operatorname{lpp}=\left\{y_{1}, r^{2}, y_{2} r, p^{2}, x_{1}^{2}\right\}$

Segment: $V_{1}$

Description: Point $P_{1}$

Basis: (infinite solutions, corresponding to degenerate triangles)

$$
B_{8}=\left\{y_{1}, r^{2}-2 r, y_{2} r-2 y_{2}, p^{2}, x_{1}^{2}-x_{2}^{2}-y_{2}^{2}+2 x_{2}-1\right\}
$$


9. Segment with lpp $=\left\{y_{2}, r^{2}, p^{2}, y_{1} p, x_{1}^{2}\right\}$

Segment: $V_{2}$

Description: Point $P_{2}$

Basis: (infinite solutions, corresponding to degenerate triangles)

$$
B_{9}=\left\{y_{2}, r^{2}-2 r+1, p^{2}-1, y_{1} p+y_{1}, x_{1}^{2}+y_{1}^{2}-x_{2}^{2}+2 x_{2}-1\right\}
$$

\subsection{Discussion and formulation of the generalized theorem}

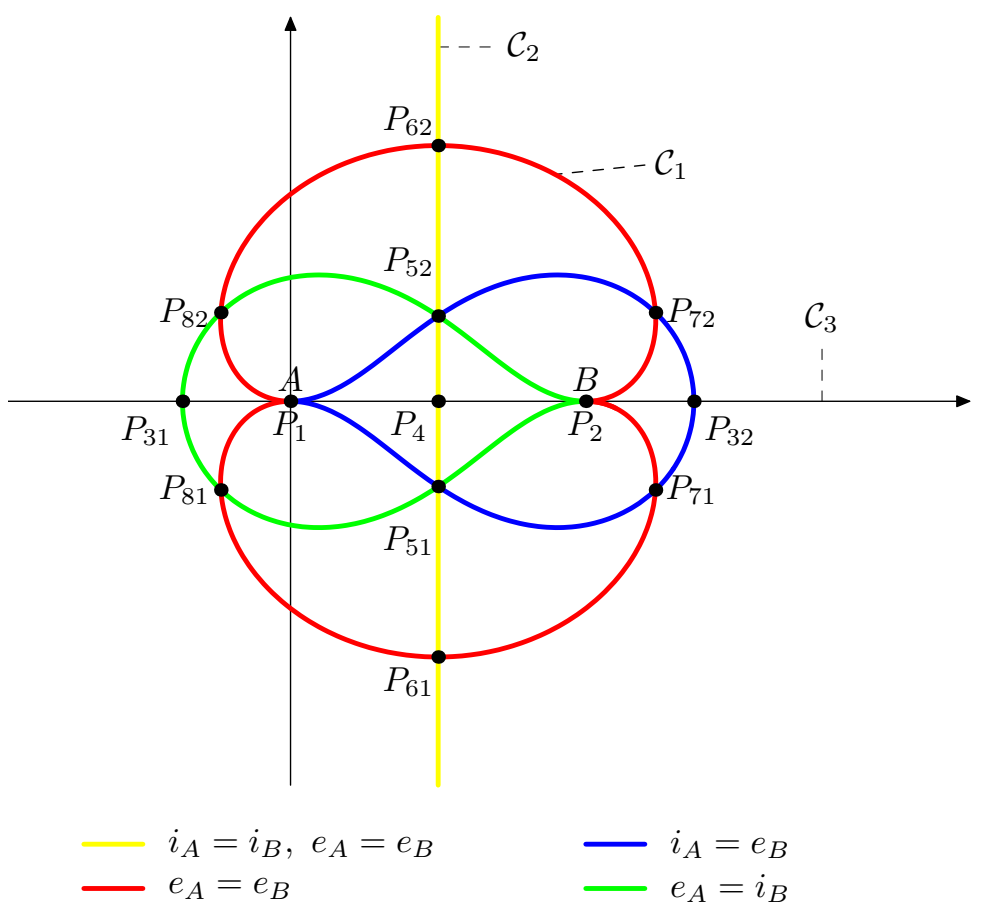

Figure 3: Problem 1. Curves $\mathcal{C}_{1}, \mathcal{C}_{2}, \mathcal{C}_{3}$ and special points

- Segment 1: of the Gröbner cover proves that the thesis does not hold in general, except for triangles with vertex $C$ placed on the three curves $\mathcal{C}_{1}$, $\mathcal{C}_{2}$ and $\mathcal{C}_{3}$ (see Figure 3). For the points inside these curves, system (3) has always some solution. Let us discuss which kind of solutions exist on these curves.

- Segment 2: It has two components: 
1) For $a=1 / 2$, vertex $C$ is on the bisector of side $A B$ (so that the triangle $A B C$ is isosceles), leaving out the points $P_{51}, P_{52}, P_{61}, P_{62}, P_{4}$. Specializing the basis on this branch (setting $a=1 / 2$ ) yields to

$$
\begin{aligned}
B_{21}= & \left\{-p-r+1,\left(4 b^{2}-3\right) y_{2}+4(b) r,\left(4 b^{2}-3\right) x_{2}+2 r,\right. \\
& \left(4 b^{2}-3\right) y_{1}+(4 b) r,\left(4 b^{2}-3\right) x_{1}-2 r+\left(-4 b^{2}+3\right), \\
& \left.4 r^{2}-8 r+3+\left(-4 b^{2}\right)\right\}
\end{aligned}
$$

and considering the first and the last equations we have:

$$
p=1-r=(1 / 2) \pm \sqrt{1+4 b^{2}}
$$

Thus there are two solutions: In one case $p=1-r>0$, so that it holds the equality of both internal bisectors (i.e. $i_{A}=i_{B}$ ), and in the other solution it holds that $p=1-r<0$, corresponding to the case $e_{A}=e_{B}$, which is also obvious from the first equality, by symmetry.

Thus, on this part of the segment the two inner bisectors are equal, as well as the two outer ones. This corresponds to the classical SteinerLehmus Theorem, enlarging it with the coincidence of the outer bisectors too.

2) $V_{8}$, containing the pair of points $P_{81}$ and $P_{82}$. Here we have

\begin{tabular}{|l|l|l|}
\hline Point & $(p, 1-r)$ & Bisectors \\
\hline$P_{81}, P_{82}$ & $(-0.3819659526,-1.272019650)$ & $e_{A}=e_{B}$ \\
& $(-0.3819659526,1.272019650)$ & $e_{A}=i_{B}$ \\
\hline
\end{tabular}

- Segment 5: It contains the two real points $P_{51}, P_{52}$, and for each one there are four solutions, as it is clear by observing the values of $p$ and $1-r$ at each of the solutions.

\begin{tabular}{|l|l|l|}
\hline Point & $(p, 1-r)$ & Bisectors \\
\hline$P_{51}, P_{52}$ & $(0.5773502693,0.5773502693)$ & $i_{A}=i_{B}$ \\
& $(0.5773502693,-0.577350269)$ & $i_{A}=e_{B}$ \\
& $(-0.5773502693,0.5773502693)$ & $e_{A}=i_{B}$, \\
& $(-0.5773502693,-0.5773502693)$ & $e_{A}=e_{B}$ \\
\hline
\end{tabular}

- Segment 6: contains the two real points $P_{61}, P_{62}$, and for each one there is a unique solution corresponding to $i_{A}=i_{B}$ (as it can be checked 
by actually solving the system associated to segment 6). We observe -as remarked at the end of section 1 - that, although at these points there should be -by symmetry- another solution, related to the equality $e_{A}=e_{B}$, it is actually missing, because both external angle bisectors become infinite. This is the reason why, even if the lpp on this segment 6 is equal to the lpp on segment 3, this common lpp appears in different segments. We will see below that, in the curve described in segment 3 , in the neighborhood of $P_{61}$ and $P_{62}$, we have the equality $e_{A}=e_{B}$, instead of $i_{A}=i_{B}$.

\begin{tabular}{|l|l|l|}
\hline Point & $(p, 1-r)$ & Bisectors \\
\hline$P_{61}, P_{62}$ & $(1,1)$ & $i_{A}=i_{B}$ \\
\hline \hline
\end{tabular}

- Segment 7: $V_{7}$, containing the pair of points $P_{71}$ and $P_{72}$. Here we have

\begin{tabular}{|l|l|l|}
\hline Point & $(p, 1-r)$ & Bisectors \\
\hline$P_{71}, P_{72}$ & $(-1.272019650,-0.381965976)$ & $e_{A}=e_{B}$ \\
& $(1.272019650,-0.381965976)$ & $i_{A}=e_{B}$ \\
\hline
\end{tabular}

- Segment 3: This segment contains all the points of the curve $\mathcal{C}_{1}$ except the special points. There is a unique solution on each point of the curve, and so only one equality between one bisector of $A$ and one bisector of $B$ can happen. The kind of solution cannot change, by continuity, on the curve except when the curve reaches a special point. The reason is that in the changing points one needs to have equality of more bisectors and this can only occur in some special segment. So we only need to determine the color (i.e. the kind of solution) -in Figure 3- of a single point of the curve between special points.

We can proceed, then, by choosing some simple vertical lines, determining its intersection with the curve and computing in each case the correspondent bisectors. For instance, for the lines $x=0$ and $x=1$ (which determine quite a few of the pieces of the curve; a similar procedure should be performed on the remaining parts) we obtain the following systems of equations for the intersections:

$$
\begin{aligned}
& \left\{\begin{array}{l}
a=0 \\
b^{2}\left(3 b^{4}-4 b^{3}+5 b^{2}-4 b+1\right)\left(3 b^{4}+4 b^{3}+5 b^{2}+4 b+1\right)
\end{array}\right. \\
& \left\{\begin{array}{l}
a=1 \\
b^{2}\left(3 b^{4}-4 b^{3}+5 b^{2}-4 b+1\right)\left(3 b^{4}+4 b^{3}+5 b^{2}+4 b+1\right)
\end{array}\right.
\end{aligned}
$$


We do not consider the solutions $(a, b)=(0,0)$ and $(a, b)=(1,0)$, as they correspond to degenerate triangles. Substituting the solutions of these systems into the basis $B_{3}$ one can determine the pair $(p, 1-r)$ for each of the points, thus determining which bisectors are equal at the corresponding point. We set a red color if $e_{A}=e_{B}$, blue color if $i_{A}=e_{B}$ and green if $e_{A}=i_{B}$. The possibility $i_{A}=i_{B}$ never occurs on $\mathcal{C}_{1}^{*}$. The following table gives the color assigned in Figure 3 to some points of the curve

\begin{tabular}{|l|l|l|l|}
\hline Point & Branch & $(p, 1-r)$ & Bisectors \\
\hline$(0, .7013671986)$ & $P_{62}-P_{82}$ & $(-.7013671074,-1.221439949)$ & $e_{A}=e_{B}$ \\
\hline$(0, .4190287818)$ & $P_{52}-P_{82}$ & $(-.4190287676,1.08424403111)$ & $e_{A}=i_{B}$ \\
\hline$(0,-.4190287818)$ & $P_{51}-P_{81}$ & $(-.4190287676,1.08424403111)$ & $e_{A}=i_{B}$ \\
\hline$(0,-.7013671986)$ & $P_{61}-P_{81}$ & $(-.7013671074,-1.221439949)$ & $e_{A}=e_{B}$ \\
\hline$(1, .7013671986)$ & $P_{62}-P_{92}$ & $(-1.221530232,-0.701371729)$ & $e_{A}=e_{B}$ \\
\hline$(1, .4190287818)$ & $P_{52}-P_{92}$ & $(1.084234608,-0.419025294)$ & $i_{A}=e_{B}$ \\
\hline$(1,-.4190287818)$ & $P_{51}-P_{91}$ & $(1.084234608,-0.419025294)$ & $i_{A}=e_{B}$ \\
\hline$(1,-.7013671986)$ & $P_{61}-P_{91}$ & $(-1.221530232,-0.701371729)$ & $e_{A}=e_{B}$ \\
\hline
\end{tabular}

- Segments 4, 8, 9: These three segments correspond to degenerate triangles. Here there are infinite solutions, as the lengths of the bisectors are not defined. We need not to analyze what happens exactly over them.

Obviously all the properties concerning the shape of the curve and the special points and colors are easily transformed if scaling the distance $\overline{A B}$.

In summary, we have thus proved the following

Theorem 3.2 (Generalized Steiner-Lehmus). Let $A B C$ be a triangle and $i_{A}$, $e_{A}, i_{B}, e_{B}$, the lengths of the inner and outer bisectors of the angles $A$ and $B$. Then, considering the conditions for the equality of some bisector of $A$ and some bisector of $B$ the following excluding situations occur:

1. The triangle $A B C$ is degenerate (i.e. $C$ is aligned with $A$ and $B$ );

2. $A B C$ is equilateral and then $i_{A}=i_{B}$, whereas $e_{A}$ and $e_{B}$ become infinite, $\left(P_{61}, P_{62}\right)$; 
3. Point $C$ is in the center of an equilateral triangle, and then $i_{A}=i_{B}=$ $e_{A}=e_{B},\left(P_{51}, P_{52}\right)$

4. The triangle is isosceles, but not of the special form of cases 2. or 3. and then $i_{A}=i_{B} \neq e_{A}=e_{B}$, (ordinary Theorem);

5. $\frac{\overline{A C}}{\overline{A B}}=\sqrt{\frac{1+\sqrt{5}}{2}}, \frac{\overline{B C}}{\overline{A B}}=\frac{3-\sqrt{5}}{2}$, and then $e_{A}=e_{B}=i_{A},\left(P_{71}, P_{72}\right)$;

6. $\frac{\overline{A C}}{\overline{A B}}=\frac{3-\sqrt{5}}{2}, \frac{\overline{B C}}{\overline{A B}}=\sqrt{\frac{1+\sqrt{5}}{2}}$, and then $e_{A}=e_{B}=i_{B},\left(P_{81}, P_{82}\right)$;

7. $C$ lies in the curve of degree 10 relative to points $A$ and $B$ (case of curve $\mathcal{C}_{1}$ ) passing through all the special points above but is none of these points, and then only one of the following possibilities happen: either $e_{A}=e_{B}$ or $i_{A}=e_{B}$ or $e_{A}=i_{B}$, depending on the piece of the curve (see Figure 3, the color representing which of these situations occur);

8. None of the above cases occur, and then no bisector of $A$ is equal to no bisector of $B$.

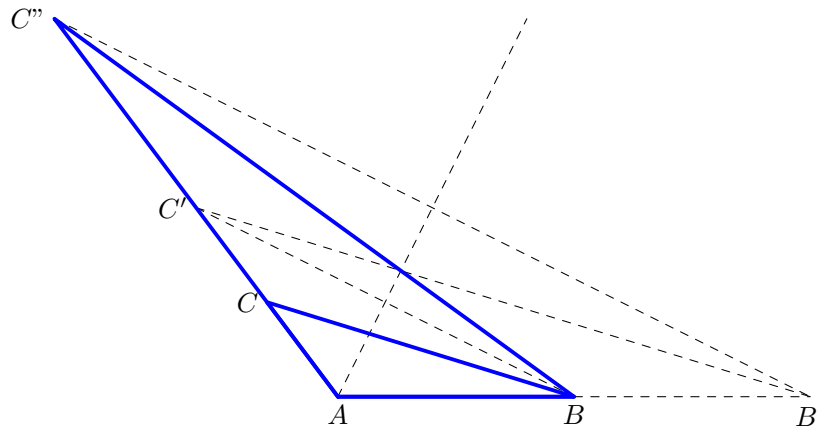

Figure 4: Transformation of coordinates 


\subsection{Bisectors at vertex $C$}

So far we have studied the equality of bisectors at the fixed points $A, B$ of the given triangle. For the sake of completeness, it could be of some interest to analyze the conditions for one bisector of the fixed point $A$ to be equal to one bisector of the moving point $C$. Obviously, a similar task could be undertaken regarding the couple $B, C$.

We can deal with the equality of bisectors for vertices $A, C$ as in the precedent case of the vertices $A, B$, by computing the solutions of the corresponding polynomial system using the Gröbner cover. The computations are completely similar to those of the precedent section and, thus, we do not include here the details. See Figure 5 for a graphic representation of the output.

Yet, this issue can be also solved by a transformation of the previous solution for the equality of bisectors at the fixed points $A, B$. In fact: each point $C$ of the solution to this problem corresponds to a triangle $A B C$ (see Figure 4), where one bisector of $A$ is equal to one bisector of $B$, with $\overline{A B}=1$. Considering a parallel to the line $B C$ one can form a similar triangle $A B^{\prime} C^{\prime}$ with $\overline{A C^{\prime}}=1$. Making a symmetry over the inner bisector of $A$ will lead to a new triangle $A B C^{\prime \prime}$ with one bisector of $A$ equal to one bisector of $C^{\prime \prime}$ and $\overline{A B}=1$ that corresponds to the requirements of the new problem. This yields a transformation of $C$ into $C^{\prime \prime}$ that will conserve the direction $\overrightarrow{A C}$ parallel to $\overrightarrow{A C^{\prime \prime}}$, but having inverse lengths. Thus, setting $C=(a, b)$ and $C^{\prime \prime}=\left(a^{\prime}, b^{\prime}\right)$ the transformation is

$$
\left\{\begin{array} { l } 
{ a ^ { \prime } = \frac { a } { a ^ { 2 } + b ^ { 2 } } } \\
{ b ^ { \prime } = \frac { b } { a ^ { 2 } + b ^ { 2 } } }
\end{array} \quad \left\{\begin{array}{l}
a=\frac{a^{\prime}}{a^{\prime 2}+b^{\prime 2}} \\
b=\frac{b^{\prime}}{a^{\prime 2}+b^{\prime 2}}
\end{array}\right.\right.
$$

Substituting the transformation into the curves obtained in the precedent section and eliminating the denominator $\left(a^{2}+b^{2}\right)^{s}$ (where $s$ depends on the curve), leads to the transformed curves

$$
\begin{array}{rlr}
\mathcal{C C}_{1}= & \mathbb{V}\left(a^{8} b^{2}+4 a^{6} b^{4}+6 a^{4} b^{6}+4 a^{2} b^{8}+b^{1} 0-4 a^{8}-18 a^{6} b^{2}-30 a^{4} b^{4}\right. \\
& -22 a^{2} b^{6}-6 b^{8}+8 a^{7}+28 a^{5} b^{2}+32 a^{3} b^{4}+12 a b^{6}+16 a^{6}+31 a^{4} b^{2} \\
& +14 a^{2} b^{4}-b^{6}-64 a^{5}-100 a^{3} b^{2}-36 a b^{4}+76 a^{4}+94 a^{2} b^{2}+14 b^{4} \\
& \left.-40 a^{3}-44 a b^{2}+8 a^{2}+9 b^{2}\right) \\
\mathcal{C C}_{2}= & \mathbb{V}\left(a^{2}-2 a+b^{2}\right), \quad \mathcal{C C}_{3}=\mathbb{V}(b)
\end{array}
$$

where the curves $\mathcal{C C}_{i}$ correspond to $\mathcal{C}_{i}$ and the points $Q_{i j}$ to $P_{i j}$. All of them are represented in Figure 5. 


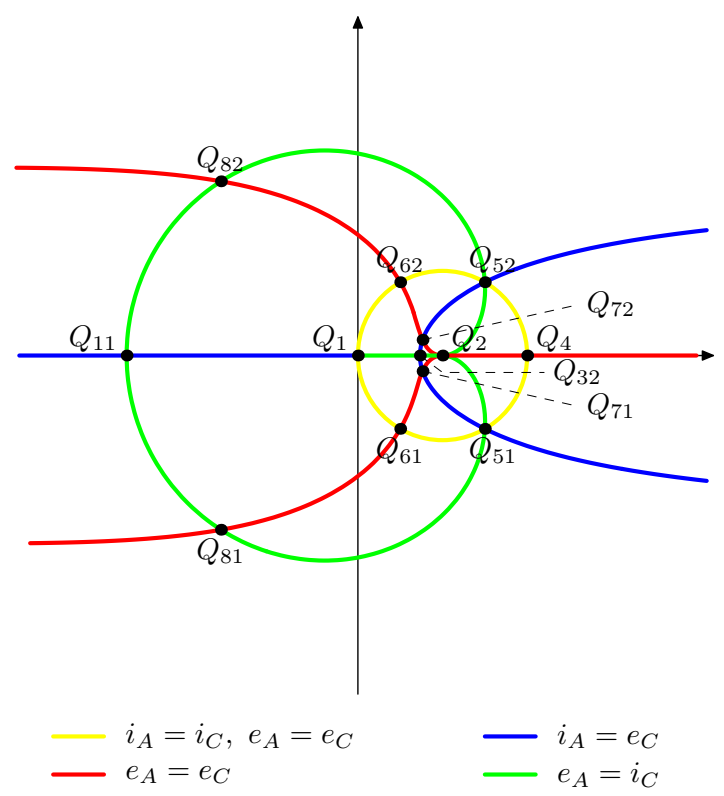

Figure 5: Problem 2. Curves $\mathcal{C C}_{1}, \mathcal{C C}_{2}$

\section{Conclusions}

As stated in the Introduction, neither the Gröbner cover method, nor the application of tools for discussing parametric systems of equations to the automatic discovery of geometric statements or the automatic discovery of the Steiner-Lehmus generalization, are new ideas. What is new in this paper is the application of the Gröbner cover tool to automatic discovery, in the context of the Steiner-Lehmus theorem.

The very relevant case distinction of subsection 3.1 (describing the pieces of the involved curves where the equality holds for internal/internal, internal/external or external/external bisectors), as graphically displayed in Figures 3 and 5 , is a natural output when using the Gröbner cover method, but unfeasible (in an automatic manner) for the previous approaches to this problem, where this case distinction was achieved by performing different tests at some selected points in the curves.

It is probably true that such case distinction could also have been achieved using other algorithms related to comprehensive Gröbner systems. Now, roughly speaking, there are two kinds of algorithms for discussing parametric 
ideals: Comprehensive Gröbner Systems (CGS) (Weispfenning [22], SuzukiSato [20], Nabeshima [15], Kapur-Sun-Wang [6], Montes [12]) and Canonical algorithms (the Montes-Wibmer Gröbner cover [14] and its predecessor MCCGS [10]). The reference [23] contains an algorithm which is canonical only on some restricted sense (the output just depends on the ideal and monomial order, but the produced segments are not disjoint and their description is not compact). Currently, among the CGS methods, we have to consider only KSW (Kapur-Sun-Wang), as it is much faster, produces disjoint segments and reduced bases and also produces much less segments. Other methods can be considered as previous attempts, but have now become obsolete.

Concerning canonical methods, the current one is the Gröbner cover (GC). KSW (Kapur-Sun-Wang) is actually used inside GC as its starting CGS step, and then it suitably transforms its output to obtain the canonical Gröbner cover.

Comparing KSW with GC, concerning the Steiner-Lehmus problem, we have the following

\begin{tabular}{|l|c|c|c|}
\hline Algorithm & Number of segments & Time(sec) & $\begin{array}{c}\text { For use in } \\
\text { grobcov (sec.) }\end{array}$ \\
\hline KSW & 14 & 0.39 & 1.07 \\
\hline GC & 9 & - & 3.42 \\
\hline
\end{tabular}

Computations have been done with a MacBook Pro, Version 10.7.4, with 2.3 GHz Intel Core i7 processor and 4GB $1600 \mathrm{MHz}$ DDR3 memory.

The third column (KSW time alone) refers to simply applying KSW to the given ideal. The corresponding segments are represented as difference of varieties, with no further requirements, and thus the obtained segments do not necessarily correspond to those in the GC method (see below for further details). In the last column we display the time needed for using KSW inside grobcov, requiring, first homogenizing the given ideal, then computing KSW, then grouping segments by lpp, then dehomogenizing the bases and, finally transforming the segments into canonical P-representation. This corresponds to the first step of grobcov, as described above.

In some detail, it turns out that 4 segments of KSW of the homogenized ideal are grouped into segment 2 of GC, two segments of KSW are grouped into segment 3 of GC and two more into segment 4 . The remaining KSW segments are also single segments in GC.

We believe that our work in this paper exemplifies the potential of the Gröbner cover method in the automatic discovery of geometric properties. 
Its complete automatization and implementation in this context is a future task, in which we are actually working. We refer the interested reader to [11] for a downloadable version of the current library grobcov.lib of algorithms, that will be part of the Singular [19] program in its next release.

\section{Aknowledgements}

Antonio Montes was partially supported by the Spanish "Ministerio de Ciencia y Tecnología" under the project MTM2009-07242, by the Generalitat de Catalunya under the project 2009SGR1040, and by the ESF EUROCORES programme EuroGIGA - ComPoSe IP04 - MICINN Project EUIEURC-2011-4306. Tomás Recio was partially supported by the Spanish "Ministerio de Economía y Competitividad" and by the European Regional Development Fund (ERDF), under the Projects MTM2008-04699-C03-03 and MTM2011-25816-C02- 02.

\section{References}

[1] F. Botana, Symbolic Computation and Education, chap. Bringing more intelligence to dynamic geometry by using symbolic computation, World Scientific, 2007, pp. 136-150.

[2] F. Botana, J. L. Valcarce, A software tool for the investigation of plane loci, Mathematics and Computers in Simulation 61 (2) (2003) 139-152.

[3] C. Chen, J. H. Davenport, M. Moreno-Maza, B. Xia, R. Xiao, Computing with semi-algebraic sets represented by triangular decomposition, in: A. Leykin (ed.), Proceedings of ISSAC'2011, ACM Press, New York, 2011.

[4] D. Cox, J. Little, D. O'Shea, Ideals, varieties and algorithms, $3^{\text {rd }}$ ed., Springer, New-York, 2007.

[5] G. Dalzotto, T. Recio, On protocols for the automated discovery of theorems in elementary geometry, Journal of Automated Reasoning 43 (2) (2009) 203-236.

[6] D. Kapur, Y. Sun, D. Wang, A new algorithm for computing comprehensive Gröbner systems, in: Proceedings of ISSAC'2010, ACM Press, New York, 2010. 
[7] R. Losada, T. Recio, J. L. Valcarce, Sobre el descubrimiento automático de diversas generalizaciones del teorema de Steiner-Lehmus, Boletín de la Sociedad Puig Adam 82 (2009) 53-76.

[8] R. Losada, T. Recio, J. L. Valcarce, On the automatic discovery of Steiner-Lehmus generalizations, in: J. Richter-Gebert, P. Schrek (eds.), Proceedings of ADG'2010, München, 2010.

[9] R. Losada, T. Recio, J. L. Valcarce, Equal bisectors at a vertex of a triangle, in: B. Murgante, et al. (eds.), Proceedings of ICCSA'2011, vol. 6785 of Lecture Notes in Computer Science, Springer, Berlin, 2011.

[10] M. Manubens, A. Montes, Minimal canonical comprehensive Gröbner systems, Journal of Symbolic Computation 44 (5) (2009) 463-478.

[11] A. Montes, http://www-ma2.upc.edu/ montes/, personal web page.

[12] A. Montes, A new algorithm for discussing Gröbner bases with parameters, Journal of Symbolic Computation 22 (2002) 183-208.

[13] A. Montes, T. Recio, Automatic discovery of geometry theorems using minimal canonical comprehensive Gröbner systems, in: Proceedings of ADG'2006, vol. 4869 of Lecture Notes in Artificial Intelligence, Springer, 2007.

[14] A. Montes, M. Wibmer, Gröbner bases for polynomial systems with parameters, Journal of Symbolic Computation 45 (2010) 1391-1425.

[15] K. Nabeshima, A speed-up of the algorithm for computing comprehensive Gröbner systems, in: Proceedings of ISSAC'2007, ACM Press, New York, 2007.

[16] T. Recio, P. Vélez, Automatic discovery of theorems in elementary geometry, Journal of Automated Reasoning 23 (1999) 63-82.

[17] T. Recio, P. Vélez, Numerical and symbolic ccientific computing: progress and prospects, chap. An Introduction to automated discovery in geometry through symbolic computation, Texts and Monographs in Symbolic Computation, Springer, Wien, 2011, pp. 257-271.

[18] T. Sillke, http://www.mathematik.uni-bielefeld.de/ sillke/PUZZLES/ steiner-lehmus. 
[19] Singular, http://www.singular.uni-kl.de, Computer Algebra System for polynomial computations. Center for Computer Algebra, University of Kaiserslautern, free software under the GNU General Public Licence.

[20] A. Suzuki, Y. Sato, A simple algorithm to compute comprehensive Gröbner bases, in: Proceedings of ISSAC'2006, ACM, New York, 2006.

[21] D. Wang, Elimination practice: software tools and applications, Imperial College Press, London, 2004.

[22] V. Weispfenning, Comprehensive Gröbner bases, Journal of Symbolic Computation 14 (1992) 1-29.

[23] V. Weispfenning, Canonical comprehensive Gröbner bases, Journal of Symbolic Computation 36 (2003) 669-683.

[24] W. T. Wu, X. L. Lü, Triangles with equal bisectors, People's Education Press, Beijing, 1985, in Chinese. 